\title{
Distribution of posterior corneal astigmatism and aberration before cataract surgery in Chinese patients
}

\author{
Yongxiang Jiang ${ }^{1,2} \cdot$ Yating Tang ${ }^{1,2} \cdot$ Qinghe Jing ${ }^{1,2} \cdot$ Dongjin Qian ${ }^{1,2} \cdot Y_{i}$ Lu $^{1,2}$
}

Received: 19 October 2017 / Revised: 4 March 2018 / Accepted: 30 May 2018 / Published online: 13 August 2018

(c) The Royal College of Ophthalmologists 2018

\begin{abstract}
Purpose To study the prevalence of posterior corneal astigmatism (PCA) and aberration in Chinese eyes before cataract surgery.

Methods A cross-sectional study was conducted in Eye and ENT Hospital of Fudan University, Shanghai. In all, we enrolled 1976 eligible eyes of 1976 cataract patients. The astigmatism and aberrations of anterior and posterior cornea were measured by the rotating Scheimpflug System (Pentacam HR, Oculus). Cataract was diagnosed using slit-lamp examination. Results The mean age of patients was $61.82 \pm 13.67$ years old. Mean PCA was $0.28 \pm 0.16$ (range $0-1.0$ ) D and $87.04 \%$ eyes had PCA values $<0.5 \mathrm{D}$. WTR astigmatism predominated the anterior cornea astigmatism (43.1\%), while ATR astigmatism predominated posterior $(85.4 \%)$ and total corneal astigmatism $(47.2 \%)$. We found a shift tendency of WTR to ATR with aging in anterior corneal astigmatism, while PCA remains ATR. A positive correlation between the magnitude of anterior and posterior corneal astigmatism $\left(r^{2}=0.089, P<0.001\right)$ was found, especially in WTR anterior cornea astigmatism eyes $\left(r^{2}=0.298, P<0.001\right)$. Compared with total corneal astigmatism, anterior corneal measurements overestimated WTR astigmatism by a mean of $0.24 \pm 0.13$ (D), and underestimated ATR astigmatism and oblique astigmatism in most eyes. Furthermore, anterior corneal aberrations measurements overestimated the total corneal aberration in most eyes.

Conclusions About $12.96 \%$ of eyes had PCA $\geq 0.5 \mathrm{D}$. The posterior surface remained ATR astigmatism in most cases with aging. Neglecting the posterior cornea would result in overestimation in WTR anterior corneal eyes and underestimation in ATR and oblique anterior corneal eyes. Also, the posterior corneal aberration was also essential in clinics.
\end{abstract}

\section{Introduction}

Cornea contributes over two-thirds of refractive power in the whole optical refractive power of human eyes $[1,2]$. With the rapid development and perfection of cataract surgery technique and intraocular lens (IOL) design, more accuracy is needed to measure the preoperative cornea

These authors contributed equally: Yongxiang Jiang, Yating Tang.

Yi Lu

luyieent@126.com

1 Department of Ophthalmology, Eye and ENT Hospital of Fudan University, 83 Fenyang Road, Xuhui District, 200031 Shanghai, China

2 Myopia Key Laboratory of the Health Ministry \& Visual Impairment and Reconstruction Key Laboratory of Shanghai, 200031 Shanghai, China biometry characteristics. Especially in patients with moderate or severe astigmatisms, it is critical to precisely calculate the cornea astigmatism for surgical parameters design and choosing appropriate IOL, especially of Toric IOL $[3,4]$. The total cornea characteristics comprise the anterior and posterior cornea components. A lot of publications have reported that neglecting the posterior corneal measurement may lead to deviation in the total corneal astigmatism (TCA) estimation [5-10]. Savini and Naeser [11] have found that neglecting the posterior corneal astigmatism (PCA) had the highest effect on the refractive error after Toric IOL implantation. However, studies on the overall characteristics of PCA and posterior corneal aberration in a large cohort of patients remain rare [5, 12]. Data on the status of posterior cornea are of clinical significance to the TCS assessment, cataract surgical parameters setting, and choosing appropriate IOL [1].

The Pentacam HR (Oculus Inc., Wetzlar, Germany), also called the ocular anterior segment imaging system, can provide a three-dimensional anterior segment scanning of 
the eye, and measures the anterior and posterior corneal characteristics using a rotating Scheimpflug camera [13, 14]. The excellent validity and stability of Pentacam HR to measure the anterior and posterior corneal biometry have been proved in a lot of previous studies [15-17]. So in our study, we aimed to investigate the overall distribution of astigmatism and aberrations of the posterior cornea in Chinese patients before cataract surgery using the Pentacam HR.

\section{Patients and methods}

\section{Subjects}

A cross-sectional study was carried out from June 2015 to December 2015 in Eye and ENT Hospital of Fudan University, Shanghai. It has one of the largest eye facilities in China and is located in Shanghai, Eastern China. In all, we enrolled 1976 eligible eyes of 1976 patients before cataract surgery, including congenital and age-related cataract. Participants were eligible if the patient was diagnosed with cataract using slit lamp and $+90 \mathrm{D}$ lens. Eyes with a history of ocular trauma, ocular surgery, corneal diseases, and uveitis were excluded. For patients whose both eyes met the inclusion criteria, their right eyes were included. All participants were self-identified as Han Chinese. This study adhered to the tenets of the Declaration of Helsinki. Approval was obtained from the Human Ethics Committee of Eye and ENT Hospital of Fudan University.

\section{Assessment of corneal astigmatism and aberration}

We used the Pentacam HR system (Oculus Inc., Wetzlar, Germany) to measure the anterior corneal astigmatism (ACA), PCA, and TCA and aberration. All subjects were examined by an experienced doctor (D.Q.). Patients were asked to stop wearing contact lens for more than 2 weeks before the Pentacam measurement. Briefly, the patients put their chins at right position and stared at the fixed light until a perfect alignment between the visual axial and machine sensor was obtained. Then the Pentacam scanning was performed automatically within 2-3 s. The authors had access to the information that could identify individual participants during or after data collection. We chose the data that met the quality standards for statistical analysis (quality standard showed OK in Pentacam result).

For astigmatism axis definition, we defined the ACA and TCA as "with-the-rule" (WTR) when the steep meridian of the corneal surface was between $60^{\circ}$ and $120^{\circ}$ and as "against-the-rule" (ATR) when the steep meridian of the corneal surface was between $0-30^{\circ}$ and $150-180^{\circ}$ just as previous studies classified. Furthermore, because the posterior surface is negative, PCA was defined as WTR when the steeped meridian was between $0-30^{\circ}$ and $150-180^{\circ}$, and as ATR when the steeped meridian was between $60^{\circ}$ and $120^{\circ}$. The remaining values were classified as oblique astigmatism. Moreover, we also recorded the total root mean square (RMS), lower-order aberrations RMS (LOA RMS), and higher-order aberrations RMS (HOA RMS) for the anterior, posterior, and total cornea, respectively.

\section{Statistical analysis}

We performed statistical analysis using SPSS Statistics version 22.0 (IBM/SPSS, Inc., Chicago IL). Frequency (\%) was used for the analysis of qualitative variables, and mean and standard deviation (mean $\pm \mathrm{SD}$ ) were used for the analysis of quantitative variables. The relationships between PCA and ACA, posterior corneal aberration, and age were calculated using Pearson's correlation and regression analysis. Comparisons between more than two groups were performed by one-way analysis of variance and Bonferroni test. We used the $\chi^{2}$ test to analyze the categorical data and defined statistical significance as $P \leq 0.05$.

\section{Results}

In all, 1976 eyes of 1976 cataract patients with qualified measurements were included in our study. The demographics of the study population are summarized in Table 1 . The mean value of PCA was $0.28 \pm 0.16$ (D). Most of the ACA $(92.83 \%)$ and TCA $(89.98 \%)$ values fell $<2.0 \mathrm{D}$, while most $(87.04 \%)$ of the PCA values were $\angle 0.5 \mathrm{D}$ (Fig. 1a-c). WTR astigmatism predominated the ACA (852 eyes, 43.1\%), while ATR astigmatism predominated PCA (1687 eyes, $85.4 \%$ ) and TCA (933 eyes, $47.2 \%$ ) (Fig. 1d). We also

Table 1 Demographics and mean astigmatism values of the study population

\begin{tabular}{ll}
\hline Demographics and mean astigmatism values \\
\hline Subjects (eyes) & $1976(1976)$ \\
Mean age (range) (years) & $61.82 \pm 13.67(3-100)$ \\
Gender (female:male) & $950: 1026$ \\
Mean anterior astigmatism (range) (D) & $0.92 \pm 0.65(0-4.5)$ \\
Mean posterior astigmatism (range) (D) & $0.28 \pm 0.16(0-1.0)$ \\
Mean total astigmatism (range) (D) & $1.04 \pm 0.68(0-4.7)$ \\
Mean CCT (range) ( $\mu \mathrm{m})$ & $543.14 \pm 32.81(402-666)$ \\
Anterior cornea RMS (range) $(\mu \mathrm{m})$ & $2.36 \pm 1.034(0.46-11.71)$ \\
Posterior cornea RMS (range) $(\mu \mathrm{m})$ & $0.74 \pm 0.21(0.17-3.14)$ \\
Total cornea RMS (range) $(\mu \mathrm{m})$ & $2.21 \pm 1.07(0.44-11.94)$ \\
D = diopters & \\
\hline
\end{tabular}


A

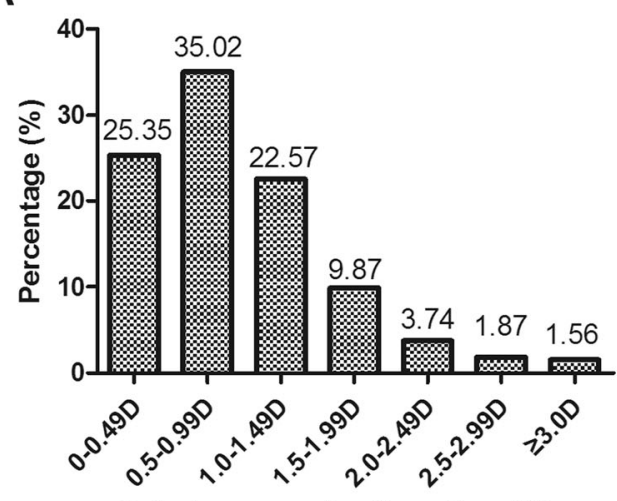

Anterior corneal astigmatism (D)

C

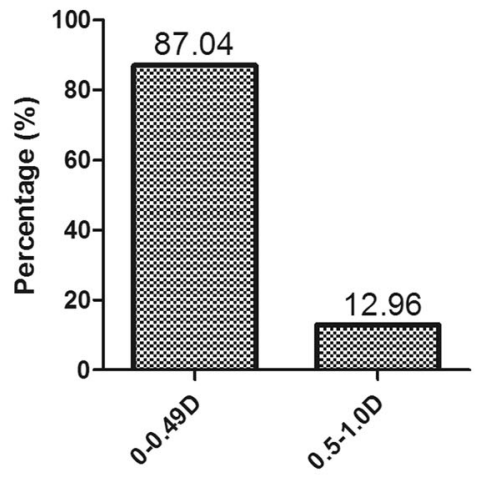

Posterior corneal astigmatism (D)

E

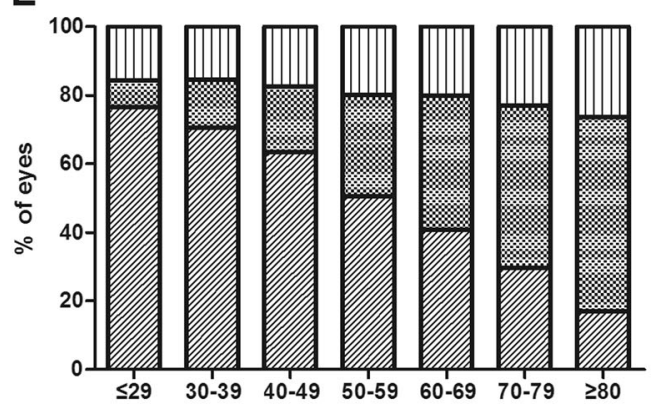

B

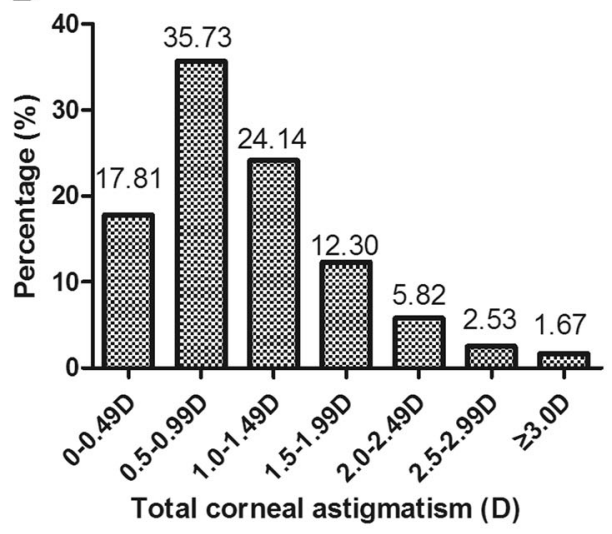

D

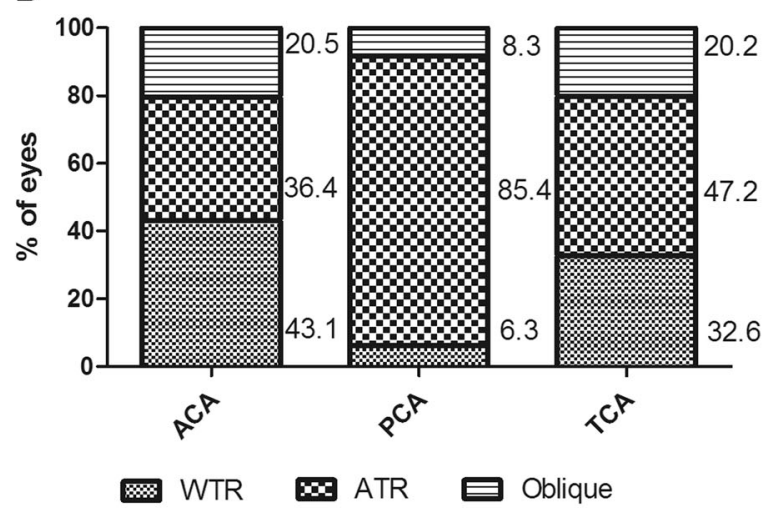

$\mathbf{F}$

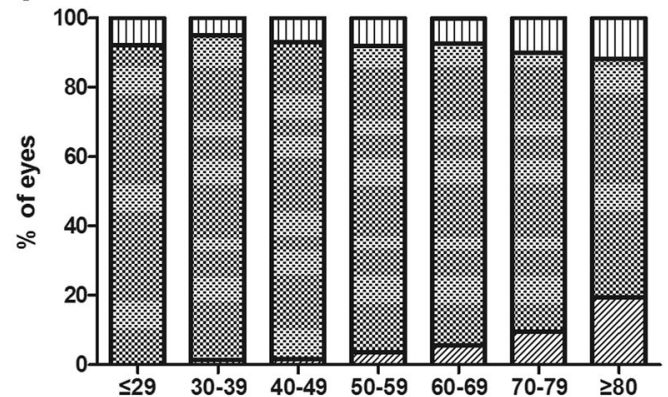

G

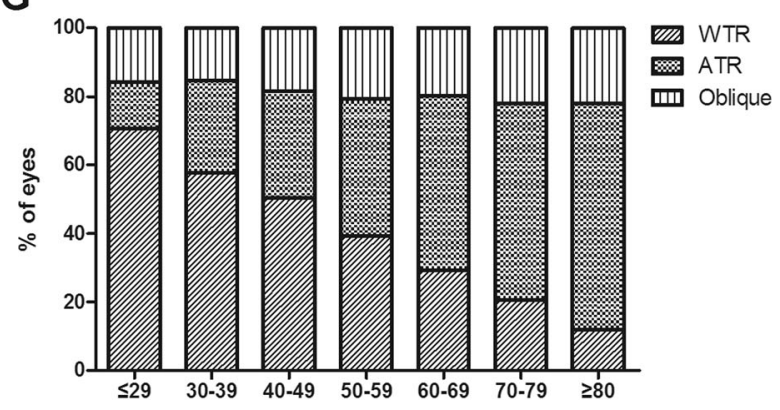

Fig. 1 a Distribution of ACA in cataract patients. b Distribution of PCA in cataract patients. c Distribution of TCA in cataract patients. d The overall distribution of anterior, posterior, and total corneal astigmatism in cataract patients. e Distribution of ACA in each age group. $\mathbf{f}$ Distribution of PCA in each age group. $\mathbf{g}$ Distribution of TCA in each age group. (D diopters, ACA anterior corneal astigmatism, PCA posterior corneal astigmatism, TCA total corneal astigmatism, WTR with the rule, ATR against the rule) 
A

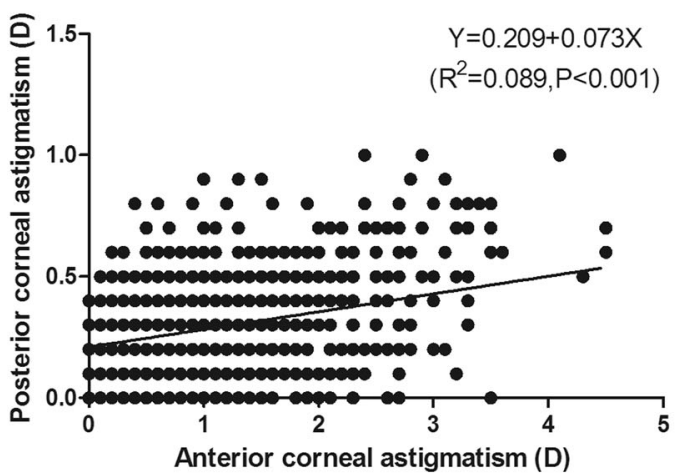

C

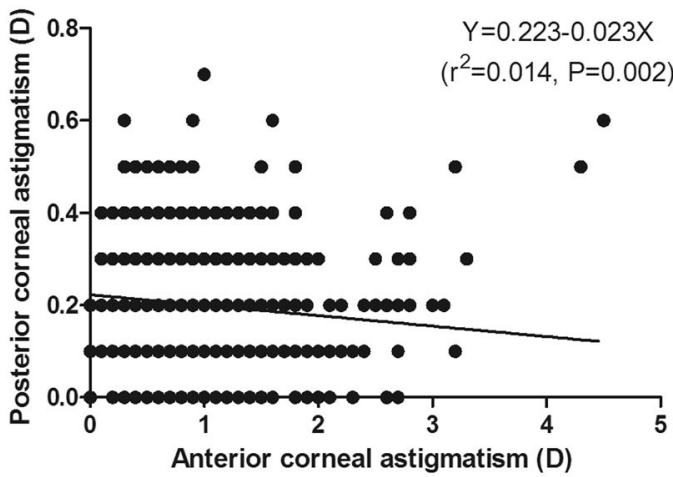

E

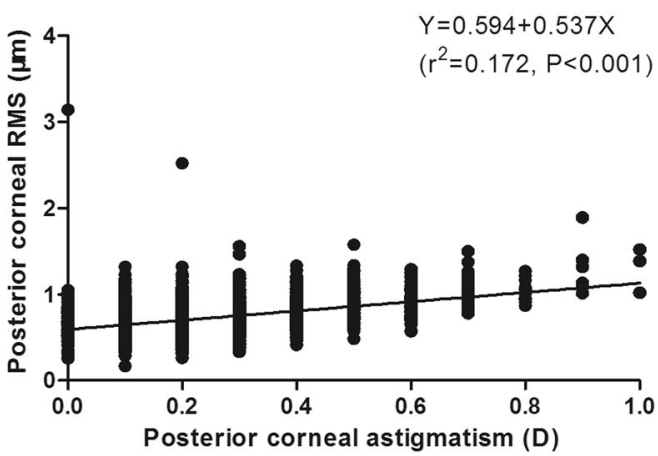

G

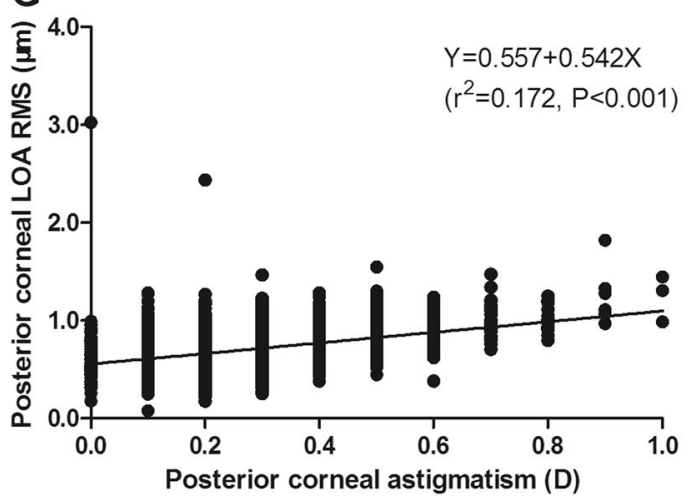

found that ATR astigmatism was the primary PCA type regardless of the ACA vector, especially in WTR astigmatism eyes.
B

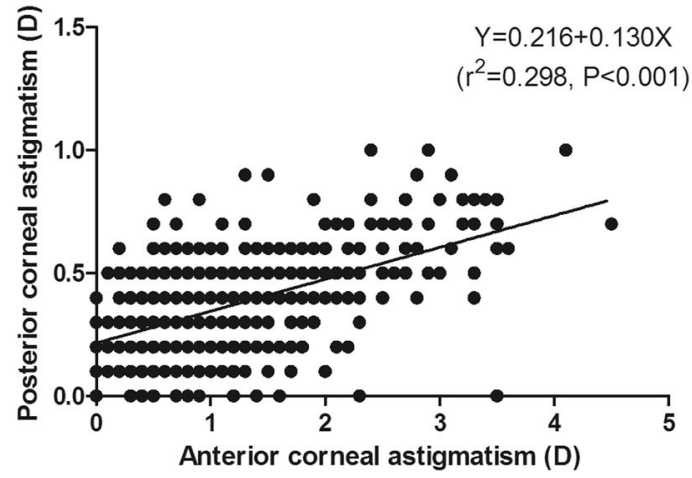

D
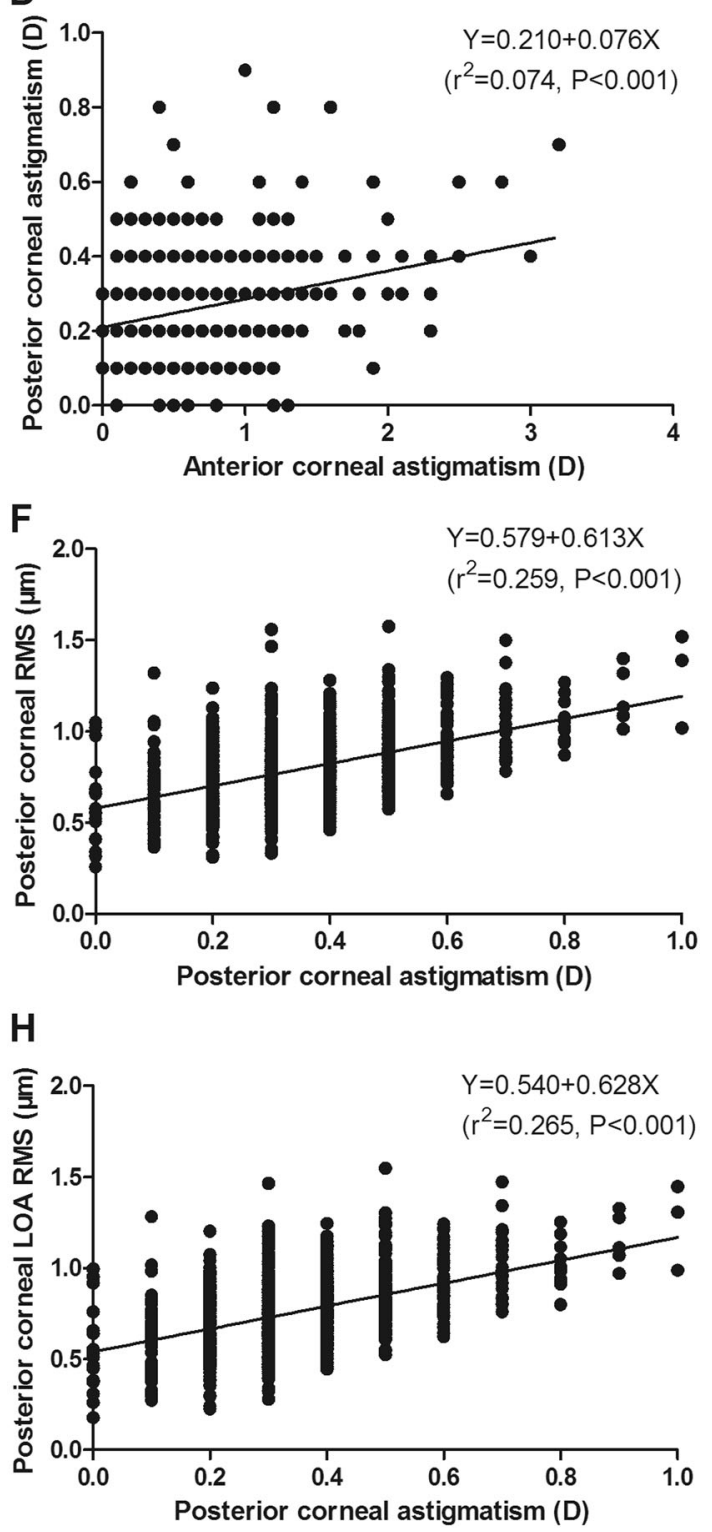

Next, we studied the corneal astigmatism in each age group. We classified the study group into the following subgroups: $\leq 29$ years (51 eyes), 30-39 years (78 eyes), 
Fig. 2 a Positive linear correlations between the magnitude of anterior and posterior corneal astigmatism $\left(r^{2}=0.089, N=1976\right)$. b Positive linear correlations between anterior and posterior corneal astigmatism in eyes with WTR anterior corneal astigmatism $\left(r^{2}=0.298, N=852\right)$. c Weak negative linear correlations between anterior and posterior corneal astigmatism in eyes with ATR anterior corneal astigmatism $\left(r^{2}\right.$ $=0.014, N=719$ ). $\mathbf{d}$ Weak positive linear correlations between anterior and posterior corneal astigmatism in eyes with oblique anterior corneal astigmatism $\left(r^{2}=0.074, N=405\right)$. e Positive linear correlations between posterior corneal astigmatism and posterior corneal aberration $\left(r^{2}=0.172, N=1976\right)$. f Positive linear correlations between posterior corneal astigmatism and posterior corneal aberration in eyes with WTR anterior corneal astigmatism $\left(r^{2}=0.259, N=852\right)$. g Positive linear correlations between posterior corneal astigmatism and posterior corneal LOA RMS $\left(r^{2}=0.172, N=1976\right)$. h Positive linear correlations between posterior corneal astigmatism and posterior corneal LOA RMS in eyes with WTR anterior corneal astigmatism $\left(r^{2}\right.$ $=0.265, N=852$ ). (WTR with the rule, ATR against the rule, RMS root mean square)

40-49 years (200 eyes), 50-59 years (394 eyes), 60-69 years (727 eyes), 70-79 years (369 eyes), and $\geq 80$ years (159 eyes). We found a tendency of increase of ATR proportion of ACA and TCA with aging (both $P<0.001$ ) (Fig. 1e, g). For PCA, we found an ATR astigmatism in most cases and a decrease of ATR astigmatism trend was found with aging $(P<0.001)$ (Fig. 1f).

We found a positive linear correlation between the magnitude of ACA and PCA $\left(r^{2}=0.089, P<0.001, N=\right.$ 1976) (Fig. 2a), especially in anterior WTR astigmatism eyes $\left(r^{2}=0.298, P<0.001, N=852\right)$ (Fig. 2b). For anterior ATR and oblique astigmatism eyes, weak negative and positive linear correlations were found between ACA and PCA, respectively $\left(r^{2}=0.014, P=0.002, N=719\right.$ for ATR and $r^{2}=0.074, P<0.001, N=405$ for oblique ACA) (Fig. 2c, d). We also analyzed the correlation between age and PCA and found weak correlation between them $\left(r^{2}=\right.$ 0.043, $P<0.001)$.

For posterior cornea aberration, we observed a positive linear correlation between PCA and posterior cornea aberration $\left(r^{2}=0.172, P<0.001, N=1976\right)$ (Fig. 2e), especially in anterior WTR eyes $\left(r^{2}=0.259, P<0.001, N=\right.$ 852) (Fig. 2f). However, little correlations were found between posterior cornea astigmatism and aberration in anterior ATR and oblique astigmatism eyes $\left(r^{2}=0.034\right.$ for ATR and $r^{2}=0.113$ for oblique, both $P<0.001$ ).

Furthermore, a positive linear correlation between PCA and posterior cornea LOA RMS was also found $\left(r^{2}=0.172\right.$, $P<0.001, N=1976$ ) (Fig. 2g), especially in WTR anterior corneal eyes $\left(r^{2}=0.265, P<0.001, N=852\right)$ (Fig. 2h). However, in ATR and oblique anterior cornea astigmatism eyes, little correlations were found between posterior cornea astigmatism and posterior cornea LOA RMS $\left(r^{2}=0.032\right.$ for ATR and $r^{2}=0.098$ for oblique, both $\left.P<0.001\right)$. We also studied the correlation between PCA and posterior cornea HOA RMS, but little correlations were found in all eyes or any vector type of ACA eyes $\left(r^{2}=0.033\right.$ for all eyes, $r^{2}=$ 0.024 for WTR eyes, $r^{2}=0.031$ for ATR eyes, and $r^{2}=$ 0.092 for oblique eyes, all $P<0.001$ ).

Compared with TCA, anterior corneal measurements overestimated WTR astigmatism by a mean of $0.24 \pm 0.13$ (D) in $64.44 \%$ eyes, underestimated ATR astigmatism by $0.39 \pm 0.20$ (D) in $89.85 \%$ eyes, and underestimated oblique astigmatism by $0.33 \pm 0.21$ (D) in $57.34 \%$ eyes (Table 2 ). For RMS measurement, anterior corneal RMS overestimated the total corneal RMS by $0.26 \pm 0.18$ (D) in $74.39 \%$ eyes and this overestimate results existed in most eyes regardless of ATR, WTR, or oblique ACA (Table 3).

\section{Discussion}

Previous studies have found that PCA measurement is significant to TCA measurement and postoperative residual refractive errors, especially in Toric IOL implanted patients. But few studies have studied the characteristics of posterior cornea in a large cohort population. So in this study, we made a detailed study of posterior cornea biometry in Chinese cataract patients. We found that the mean PCA was $0.28 \pm 0.16$ (D). There was a significant shift from WTR predominant to ATR predominant astigmatism in anterior and TCA with aging, while ATR predominated PCA in most cases. Positive linear correlations were found between the magnitude of ACA and PCA, PCA and posterior corneal RMS, and PCA and posterior corneal LOA RMS, especially in anterior WTR eyes. Neglecting the PCA would overestimate the TCA in anterior WTR astigmatism eyes, and underestimate the TCA in anterior ATR and oblique astigmatism eyes. Furthermore, neglecting PCA also causes inaccurate measurement of corneal aberrations. To our knowledge, our study is the largest cohort to study the distribution of PCA characteristics especially the posterior corneal aberrations at present.

The magnitude of PCA was first measured by Dunne and Royston [18-20] using the Purkinje images in the beginning of 1990s and by Ho [5, 7] and Koch [6] using a rotating Scheimpflug imaging. Up to now, a lot of studies have measured the PCA using various methods including the Purkinje images, Scheimpflug photography in six fixed meridians, and rotating Scheimpflug imagings [5-7, 18, 20 23]. Studies have reported that the mean values of PCA range from 0.25 to $0.78 \mathrm{D}$. Koch [6] also found that $9 \%$ of eyes had astigmatism $>0.50 \mathrm{D}$. In our study, the mean magnitude of PCA was $0.28 \pm 0.16$ (range $0-1.0$ ) (D) and $12.96 \%$ eyes had PCA $\geq 0.5 \mathrm{D}$, which was similar to previous studies, suggesting the relatively stable magnitude of PCA. From our and previous studies on PCA magnitude, we could come to the conclusion that the PCA range is $0-1.0 \mathrm{D}$, and the mean value was about $0.3 \mathrm{D}$ with ATR 
Table 2 Anterior corneal astigmatism-estimated total corneal astigmatism distribution

\begin{tabular}{|c|c|c|c|c|c|c|}
\hline \multirow{2}{*}{$\begin{array}{l}\text { Types of anterior } \\
\text { CA }\end{array}$} & \multicolumn{2}{|c|}{ Overestimated } & \multicolumn{2}{|c|}{ Underestimated } & \multirow{2}{*}{$\begin{array}{l}\text { No influence } \\
N(\%)\end{array}$} & \multirow{2}{*}{$\begin{array}{l}\text { Total } \\
N(\%)\end{array}$} \\
\hline & $N(\%)$ & $\begin{array}{l}\text { Mean } \pm \text { SD } \\
\text { (D) }\end{array}$ & $N(\%)$ & $\begin{array}{l}\text { Mean } \pm \text { SD } \\
\text { (D) }\end{array}$ & & \\
\hline WTR & 549 (64.44) & $0.24 \pm 0.13$ & $193(22.65)$ & $0.24 \pm 0.22$ & $110(12.91)$ & $852(100)$ \\
\hline ATR & $34(4.73)$ & $0.21 \pm 0.17$ & $646(89.85)$ & $0.39 \pm 0.20$ & $39(5.42)$ & 719 (100) \\
\hline Oblique & $59(14.57)$ & $0.16 \pm 0.11$ & $294(72.59)$ & $0.27 \pm 0.17$ & $52(12.84)$ & 405 (100) \\
\hline Total & $642(32.49)$ & $0.23 \pm 0.13$ & $1133(57.34)$ & $0.33 \pm 0.21$ & $201(10.17)$ & 1976 (100) \\
\hline
\end{tabular}

${ }^{*} C A$ corneal astigmatism, WTR with the rule, ATR against the rule, $D$ diopters

\begin{tabular}{|c|c|c|c|c|c|c|}
\hline \multirow{2}{*}{$\begin{array}{l}\text { Types of anterior } \\
\text { CA* }^{*}\end{array}$} & \multicolumn{2}{|l|}{ Overestimated } & \multicolumn{2}{|c|}{ Underestimated } & \multirow{2}{*}{$\begin{array}{l}\text { No influence } \\
N(\%)\end{array}$} & \multirow{2}{*}{$\begin{array}{l}\text { Total } \\
N(\%)\end{array}$} \\
\hline & $N(\%)$ & $\begin{array}{l}\text { Mean } \pm \text { SD } \\
(\mathrm{RMS})\end{array}$ & $N(\%)$ & $\begin{array}{l}\text { Mean } \pm \text { SD } \\
(\mathrm{RMS})\end{array}$ & & \\
\hline WTR & $710(83.34)$ & $0.32 \pm 0.18$ & $140(16.43)$ & $0.19 \pm 0.18$ & $2(0.23)$ & 852 (100) \\
\hline ATR & $476(66.20)$ & $0.20 \pm 0.18$ & $243(33.80)$ & $0.16 \pm 0.15$ & $0(0.00)$ & 719 (100) \\
\hline Oblique & $284(70.12)$ & $0.22 \pm 0.15$ & $120(29.63)$ & $0.18 \pm 0.17$ & $1(0.25)$ & 405 (100) \\
\hline Total & $1470(74.39)$ & $0.26 \pm 0.18$ & $503(25.46)$ & $0.17 \pm 0.16$ & $3(0.15)$ & $1976(100)$ \\
\hline
\end{tabular}

${ }^{*} C A$ corneal astigmatism, WTR with the rule, ATR against the rule, $R M S$ root mean square
Table 3 Anterior corneal aberration-estimated total corneal aberration distribution influenced the total cornea biometry, especially in the eyes of anterior WTR astigmatism. We suggest that TCA, besides ACA alone, should directly be measured and taken into account for surgical design before cataract surgery. Besides, for hospitals where total cornea measurements were not available, we could also roughly estimate the PCA based on the ACA values and our regression model. We also found very weak negative and weak positive correlations between ACA and PCA in anterior ATR astigmatism $\left(r^{2}=0.014, P=0.002\right)$ and oblique $\left(r^{2}=0.074, P<0.001\right)$ astigmatism eyes, respectively. However, no correlations were found between ACA and PCA in eyes with ATR anterior corneal surface in previous studies (both $P>0.05$ ) $[6,12]$. The very weak or no correlation between ACA and PCA implied that the huge error might have occurred when we estimated the PCA and TCA simply based on ACA in anterior ATR and oblique axis patients.

We also found a weak negative correlation between PCA and age $\left(r^{2}=0.045, P<0.001\right)$. In younger patients, the mean PCA was higher than the older ones. This might explain the relatively higher mean PCA magnitude in some studies [12, 25] compared with our study, as patients in these studies were younger than that of ours.

Furthermore, our study was the first to analyze the relationship between aberration and astigmatism. We found a significantly positive linear correlation between PCA and posterior corneal RMS, especially in anterior WTR astigmatism eyes. This indicated that in patients with higher PCA, the corresponding aberrations were also higher. Furthermore, we studied the LOA and HOA and found that the 
LOA RMS had significant positive linear correlation with PCA. This was not difficult to understand because LOA comprised mainly defocus and astigmatism while HOA comprised spherical aberration, coma, trefoil, secondary astigmatism, etc., according to the Zernike polynomials [26]. As HOA and LOA were important objective indexes of visual quality, neglecting the PCA would surely have a great influence on postoperative visual quality.

It is interesting that why aberrations were overestimated in most patients regardless of the different astigmatism deviations. One possible reason was that the corneal aberration comprised a lot of subgroups of aberrations except for the astigmatism alone. The correlation and accuracy of corneal aberration and PCA need to be studied in our further work. However, direct measurements of the posterior corneal characteristics were essential because of the complexity of astigmatism and aberration distribution.

The strengths of our study include the large sample size, detailed measurements of posterior corneal characteristics, correlation analysis between astigmatism and aberration. However, our study has some limitations. The higher prevalence of male patients might result in some bias in our study. Moreover, since our study was performed only with Pentacam, we should confirm our measuring results by other corneal imaging methods. However, it has been demonstrated that the device has an excellent repeatability of the corneal astigmatism and aberration measurements.

In summary, our study showed that $12.96 \%$ eyes had PCA $\geq 0.5 \mathrm{D}$ and neglecting the posterior corneal measurements tends to overestimate the TCA in WTR anterior corneal eyes and underestimate the TCAs in ATR and oblique anterior corneal eyes. Furthermore, the posterior corneal aberration was also essential that we need take into consideration when we conduct corneal astigmatism and IOL measurement in clinics.

\section{Summary}

\section{What was known before}

- Posterior corneal astigmatism is important for total corneal measurements.

\section{What this study adds}

- Neglecting the posterior corneal measurements tends to overestimate the total corneal astigmatism in WTR anterior corneal eyes and underestimate the total corneal astigmatisms in ATR and oblique anterior corneal eyes.

- For RMS measurement, anterior corneal RMS overestimated the total corneal RMS by $0.26 \pm 0.18$ (D) in
$74.39 \%$ eyes and this overestimate results existed in most eyes regardless of the anterior cornea astigmatism.

Acknowledgements The study was supported by grants for Natural Science Foundation of China (NSFC 81300745, NSFC81670835, and NSFC81600719), Shanghai Science and Technology Commission (11231200602), New One Hundred People's Plan of Shanghai Health Bureau (XBR2011056), and Visual Impairment and Reconstruction Key Laboratory of Shanghai (12DZ2260500).

\section{Compliance with ethical standards}

Conflict of interest The authors declare that they have no conflict of interest.

\section{References}

1. Kohnen T. Posterior corneal astigmatism. J Cataract Refract Surg. 2013;39:1795.

2. DelMonte DW, Kim T. Anatomy and physiology of the cornea. J Cataract Refract Surg. 2011;37:588-98.

3. Holland E, Lane S, Horn JD, Ernest P, Arleo R, Miller KM. The AcrySof Toric intraocular lens in subjects with cataracts and corneal astigmatism: a randomized, subject-masked, parallelgroup, 1-year study. Ophthalmology. 2010;117:2104-11.

4. Waltz KL, Featherstone K, Tsai L, Trentacost D. Clinical outcomes of TECNIS toric intraocular lens implantation after cataract removal in patients with corneal astigmatism. Ophthalmology. 2015;122:39-47.

5. Ho JD, Tsai CY, Liou SW. Accuracy of corneal astigmatism estimation by neglecting the posterior corneal surface measurement. Am J Ophthalmol. 2009;147:788-95.

6. Koch DD, Ali SF, Weikert MP, Shirayama M, Jenkins R, Wang L. Contribution of posterior corneal astigmatism to total corneal astigmatism. J Cataract Refract Surg. 2012;38:2080-7.

7. Ho JD, Liou SW, Tsai RJ, Tsai CY. Effects of aging on anterior and posterior corneal astigmatism. Cornea. 2010;29:632-7.

8. Savini G, Versaci F, Vestri G, Ducoli P, Naeser K. Influence of posterior corneal astigmatism on total corneal astigmatism in eyes with moderate to high astigmatism. J Cataract Refract Surg. 2014;40:1645-53.

9. Cheng LS, Tsai CY, Tsai RJ, Liou SW, Ho JD. Estimation accuracy of surgically induced astigmatism on the cornea when neglecting the posterior corneal surface measurement. Acta Ophthalmol. 2011;89:417-22.

10. Zheng T, Chen Z, Lu Y. Influence factors of estimation errors for total corneal astigmatism using keratometric astigmatism in patients before cataract surgery. J Cataract Refract Surg. 2016;42:84-94.

11. Savini G, Naeser K. An analysis of the factors influencing the residual refractive astigmatism after cataract surgery with toric intraocular lenses. Invest Ophthalmol Vis Sci. 2015;56:827-35.

12. Miyake T, Shimizu K, Kamiya K. Distribution of posterior corneal astigmatism according to axis orientation of anterior corneal astigmatism. PLoS ONE. 2015;10:e117194.

13. Barkana Y, Gerber Y, Elbaz U, et al. Central corneal thickness measurement with the Pentacam Scheimpflug system, optical lowcoherence reflectometry pachymeter, and ultrasound pachymetry. J Cataract Refract Surg. 2005;31:1729-35.

14. Kim JS, Chung SH, Joo CK. Clinical application of a Scheimpflug system for lens density measurements in phacoemulsification. $\mathrm{J}$ Cataract Refract Surg. 2009;35:1204-9. 
15. Lackner B, Schmidinger G, Skorpik C. Validity and repeatability of anterior chamber depth measurements with Pentacam and Orbscan. Optom Vis Sci. 2005;82:858-61.

16. Buehl W, Stojanac D, Sacu S, Drexler W, Findl O. Comparison of three methods of measuring corneal thickness and anterior chamber depth. Am J Ophthalmol. 2006;141:7-12.

17. Shankar H, Taranath D, Santhirathelagan CT, Pesudovs K. Anterior segment biometry with the Pentacam: comprehensive assessment of repeatability of automated measurements. J Cataract Refract Surg. 2008;34:103-13.

18. Dunne MC, Royston JM, Barnes DA. Posterior corneal surface toricity and total corneal astigmatism. Optom Vis Sci. 1991;68:708-10.

19. Dunne MC, Royston JM, Barnes DA. Normal variations of the posterior corneal surface. Acta Ophthalmol. 1992;70:255-61.

20. Royston JM, Dunne MC, Barnes DA. Measurement of posterior corneal surface toricity. Optom Vis Sci. 1990;67:757-63.
21. Prisant O, Hoang-Xuan T, Proano C, Hernandez E, Awwad ST, Azar DT. Vector summation of anterior and posterior corneal topographical astigmatism. J Cataract Refract Surg. 2002;28:1636-43.

22. Modis LJ, Langenbucher A, Seitz B. Evaluation of normal corneas using the scanning-slit topography/pachymetry system. Cornea. 2004;23:689-94.

23. Dubbelman M, Sicam VA, Van der Heijde GL. The shape of the anterior and posterior surface of the aging human cornea. Vision Res. 2006;46:993-1001.

24. Naderan M, Rajabi MT, Zarrinbakhsh P. Distribution of the anterior and posterior corneal astigmatism in eyes with keratoconus. Am J Ophthalmol. 2016;167:79-87.

25. Ueno Y, Hiraoka T, Beheregaray S, Miyazaki M, Ito M, Oshika T. Age-related changes in anterior, posterior, and total corneal astigmatism. J Refract Surg. 2014;30:192-7.

26. Campbell CE. A new method for describing the aberrations of the eye using Zernike polynomials. Optom Vis Sci. 2003;80:79-83. 